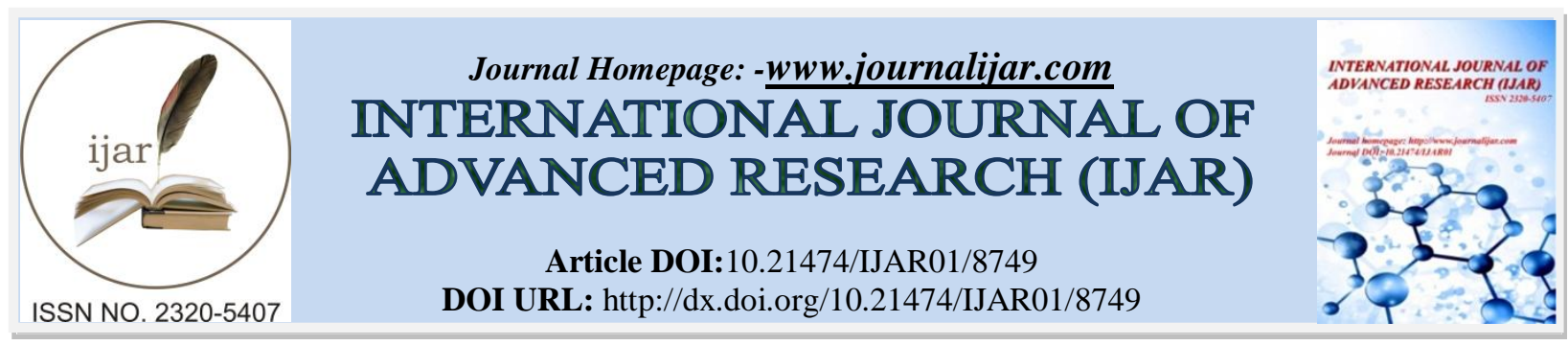

RESEARCH ARTICLE

\title{
AN HEPATIC NODULE NOT LIKE THE OTHERS.
}

Dr. M.Hajji, Dr. H.Hamri, Pr FZ L. aamrani and Pr L. Jroundi.

Unity of emergency imaging - University Hospital Ibn Sina-Rabat-Morocco.

\section{Manuscript Info}

\section{Manuscript History}

Received: 19 January 2019

Final Accepted: 21 February 2019

Published: March 2019

Key words:-

Green's function, Fick's Law, Thermal

diffusivity, Thermal conductivity.

\section{Abstract}

On a liver chronic disease, an isolated hepatic tuberculosis, though rare, must be part of the differential diagnosis especially in endemic area. we report the clinical case of Mr X , 15 years old, followed for Wilson disease arrived at the stage of cirrhosis an and in whom imaging with histological confirmation concluded at diagnosis of isolated tubercle nodule on liver of cirrhosis.

Copy Right, IJAR, 2019,. All rights reserved.

\section{Introduction:-}

Nodules on pathological liver may be related to hypo vascular CHC; a regeneration nodule; a dysplastic nodule; or more rarely an abscess.

According to the Barcelona criteria, in a cirrhotic context with nodule of 1-2cm on cirrhosis livers, an arterial enhancement associated to a washout allows a formal diagnosis only if it is found on two different imaging techniques (contrast echography, CT or MRI)

In case of atypical vascular profile or typical vascular profile but on a single imaging is an indication to perform a biopsy.

\section{Case Report}

It is about 15 years old child, followed for Wilson disease arrived at the stade of cirrhosis, and who having a notion of counting tuberculosis . he was hospitalized for a prolonged fever . clinical examination found hepatomegaly. The CRP was 59. The other biological examinations (NFS, alpha foeto-protein and immunoassay) were negative. Ultrasound finds an echogenic nodule of segment VI suggestive of hepatic angioma. The angio-CT revealed a dysmorphic liver seat of a hypo vascular nodule of segment V. MRI shows a nodule which mesure 17 $\mathrm{mm}$ hypointense in $\mathrm{t} 1$, hyper intense in T2 without restriction of diffusion, without significant enhancement after injection. The characterization of this nodule was difficult in imaging, so we realized a biopsy which revealed a granulomatous lesion with necrosis in favor of tuberculosis.

\section{Discussion:-}

Autologous liver tuberculosis is rare. The nodular liver damage in our patient is certainly due to a state of immunosuppression caused by the immunosuppressive treatment of Wilson's disease. In CT, lesions at the beginning are iso denses. They become hypodense by caseous necrosis. The injection of contrast show an annular enhancement.

In MRI, the appearance of lesions is variable. The most evocative aspect is that of hyper intense lesion at the periphery, with less intensity in the center, on the T2-weighted sequences, which enhances itself at the periphery 
after contrast injection. Cystic lesions are T1 hypo signal, and hyper T2. Hepatic histology by echo-guided biopsy or guided scan is important for diagnosis. It allows the detection of necrotic material caseous.

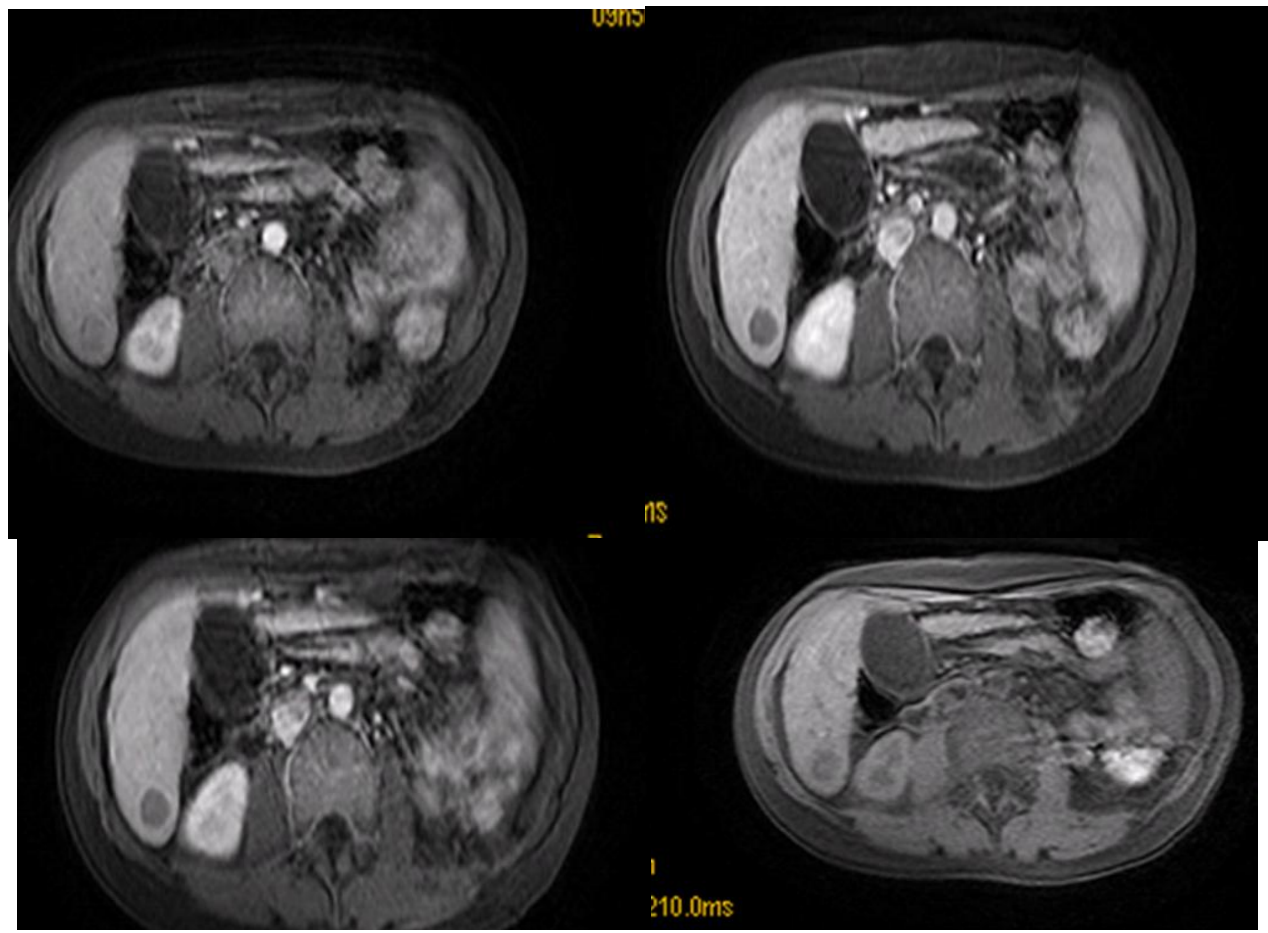

Fig 1:-MRI findings

1. Liver increased in size.

2. Presence of a segment VI nodule, measuring $17 \mathrm{~mm}$ in hypersignal $\mathrm{T} 2$ and hyposignal diffusion and in opposition of phase, RH -.

3. Homogeneous Splenomegaly.

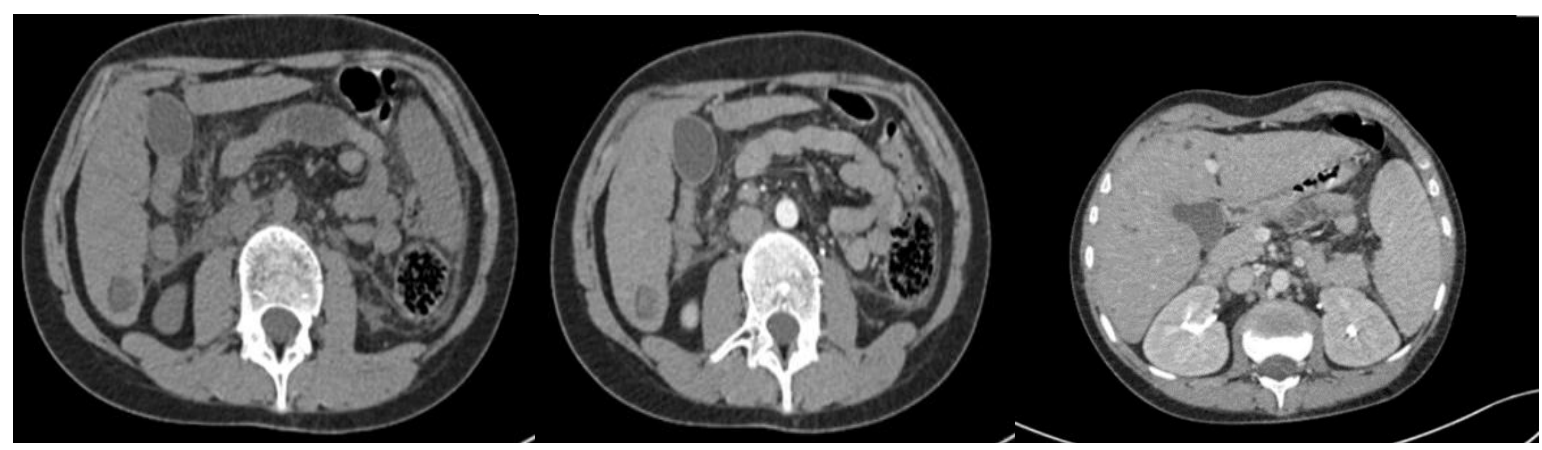

Fig 2:-CT-scan findings :

1. Liver with multiple hypodense nodules interesting all segments.

2. The largest nodule is $18 \mathrm{~mm}$, very finely rounded, hypodense in spontaneous contrast, slightly raised after injection of Contrast product at different waiting times

\section{Conclusion:-}

Isolated hepatic tuberculosis, though rare, must be part of the differential diagnosis in the presence of a mass occurring on a liver of chronic liver disease. The is necessary in case of doubt, especially if the diagnostic criteria for hepatocellular carcinoma are not met.

\section{Competing interests:-}

The authors declare that they have no competing interests. 